\title{
Application of double Laplace decomposition method for solving singular one dimensional system of hyperbolic equations
}

\author{
Hassan Eltayeb Gadain \\ Mathematics Department, College of Science, King Saud University, P. O. Box 2455, Riyadh 11451, Saudi Arabia. \\ Communicated by B. Samet
}

\begin{abstract}
In this paper, the Adomain decomposition methods and double Laplace transform methods are combined to study linear and nonlinear singular one dimensional system of hyperbolic equations. In addition, we check the convergence of double Laplace transform decomposition method applied to our problems. Furthermore, we illustrate our proposed methods by using some examples. (C)2017 All rights reserved.
\end{abstract}

Keywords: Double Laplace transform, inverse Laplace transform, system of hyperbolic equations. 2010 MSC: 35A44, 65M55.

\section{Introduction}

Many applications in sciences are modeled by linear and nonlinear partial differential equations. The hyperbolic partial differential equations as one of these applications arise in physical sciences as models of waves, such as acoustic, elastic, electromagnetic, or gravitational waves. However, it is very difficult to find explicit solutions of nonlinear partial differential equations generally. The Adomain decomposition method is the most transparent method for solutions of linear and nonlinear problem (see [3, 5, 7, 16, 17]); however, this method is involved in the calculation of complicated Adomain's polynomials which narrow down its application. Recently, many researchers and engineers have done excellent work, such as Laplace decomposition algorithm $[12,18]$. The convergence of Adomian's method has been studied by several authors $[1,2,4,6]$. The aim of this article is to find the solution of linear and nonlinear singular one dimensional system hyperbolic equations by using the combined domain decomposition techniques and double Laplace transform methods and also we study the sufficient condition of convergence of our methods. The gold of this method is that it can be used directly without using restrictive assumptions or linearization. Throughout this article, we will use the following notation. We let $L_{x} L_{t}$ be a double Laplace transform with respect to $x, t$ and let double inverse Laplace transform be defined by $\mathrm{L}_{\mathrm{p}}^{-1} \mathrm{~L}_{\mathrm{s}}^{-1}$.

Now, by calling the definitions which are given by $[8,13,14]$. The double Laplace transform of the functions $f(x, t), \frac{\partial \psi}{\partial x}, \frac{\partial^{2} \psi}{\partial x^{2}}$ and $\frac{\partial^{2} \psi}{\partial t^{2}}$ are given by 


$$
\begin{aligned}
\mathrm{L}_{x} \mathrm{~L}_{\mathrm{t}}[\mathrm{f}(\mathrm{x}, \mathrm{t})]=\mathrm{F}(\mathrm{p}, \mathrm{s}) & =\int_{0}^{\infty} e^{-p x} \int_{0}^{\infty} e^{-s t} f(x, t) d t d x \\
\mathrm{~L}_{x} \mathrm{~L}_{t}\left[\frac{\partial \psi}{\partial x}\right] & =p \psi(p, s)-\psi(0, s) \\
\mathrm{L}_{x} \mathrm{~L}_{t}\left[\frac{\partial^{2} \psi}{\partial x^{2}}(x, t)\right] & =p^{2} \psi(p, s)-p \psi(0, s)-\frac{\partial \psi}{\partial x} \\
\mathrm{~L}_{x} \mathrm{~L}_{t}\left[\frac{\partial^{2} \psi}{\partial t^{2}}(x, t)\right] & =s^{2} \psi(p, s)-s \psi(p, 0)-\psi_{t}
\end{aligned}
$$

The following basic lemma of the double Laplace transform is given and is used in this paper.

Lemma 1.1. Double Laplace transform of the non-constant coefficient second order partial derivative $x^{r} \frac{\partial^{2} \psi}{\partial t^{2}}$ and the function $x^{r} f(x, t)$ are given by

$$
L_{x} L_{t}\left(x^{r} \frac{\partial^{2} \psi}{\partial t^{2}}\right)=(-1)^{r} \frac{d^{r}}{d p^{r}}\left[s^{2} \psi(p, s)-s \psi(p, 0)-\psi_{t}\right]
$$

and

$$
L_{x} L_{t}\left(x^{r} f(x, t)\right)=(-1)^{r} \frac{d^{r}}{d p^{r}}\left[L_{x} L_{t}(f(x, t))\right]=(-1)^{n} \frac{d^{r} F(p, s)}{d p^{r}}
$$

where $r=1,2,3, \cdots$.

One can prove this lemma by applying equations, (1.1), (1.2) and (1.3). In this study we present the modified double Laplace decomposition method for solving singular one dimensional coupled system of hyperbolic equations.

\section{Statement of the problem} form

We consider a singular one dimensional system hyperbolic equations with initial conditions in the

$$
\begin{aligned}
& \frac{\partial^{2} \psi}{\partial t^{2}}-\frac{1}{x}\left(x \frac{\partial \psi}{\partial x}\right)_{x}-\varphi=f(x, t) \\
& \frac{\partial^{2} \varphi}{\partial t^{2}}-\frac{1}{x}\left(x \frac{\partial \varphi}{\partial x}\right)_{x}-\psi=g(x, t)
\end{aligned}
$$

subject to

$$
\begin{array}{ll}
\psi(x, 0)=f_{1}(x), & \psi_{t}(x, 0)=f_{2}(x), \\
\varphi(x, 0)=g_{1}(x), & \varphi_{t}(x, 0)=g_{2}(x),
\end{array}
$$

where, the linear term, $\frac{1}{x} \frac{\partial}{\partial x}\left(x \frac{\partial \psi}{\partial x}\right)$ and $\frac{1}{x}\left(x \frac{\partial \varphi}{\partial x}\right)_{x}$ are called Bessel's operators and $f(x, t), g(x, t), f_{1}(x)$, $f_{2}(x), g_{1}(x)$ and $g_{2}(x)$ are known functions. In order to obtain the solution of (2.1), we use modified double Laplace decomposition methods as follows.

Step 1: Multiplying (2.1) by $x$.

Step 2: By using Lemma 1.1 and definition of the double Laplace transform of partial derivatives for 
equations in Step 1 and single Laplace transform for initial condition, we get

$$
\begin{aligned}
\frac{d \psi(p, s)}{d p}= & \frac{1}{s} \frac{d F_{1}(p)}{d p}+\frac{1}{s^{2}} \frac{d F_{2}(p)}{d p}+\frac{1}{s^{2}} \frac{d F(p, s)}{d p} \\
& -\frac{1}{s^{2}} L_{x} L_{t}\left[\frac{\partial}{\partial x}\left(x \frac{\partial \psi}{\partial x}\right)+x \varphi\right],
\end{aligned}
$$

and

$$
\begin{aligned}
\frac{d \varphi(p, s)}{d p}= & \frac{1}{s} \frac{d G_{1}(p)}{d p}+\frac{1}{s^{2}} \frac{d G_{2}(p)}{d p}+\frac{1}{s^{2}} \frac{d G(p, s)}{d p} \\
& -\frac{1}{s^{2}} L_{x} L_{t}\left[\frac{\partial}{\partial x}\left(x \frac{\partial \varphi}{\partial x}\right)+x \psi\right],
\end{aligned}
$$

where $F_{1}(p), F_{2}(p), F(p, s), G_{1}(p), G_{2}(p)$ and $G(p, s)$ are Laplace transform of the functions $f_{1}(x)$, $f_{2}(x), f(x, t), g_{1}(x), g_{2}(x)$ and $g(x, t)$, respectively.

Step 3: By integrating both sides of (2.3) and (2.4) from 0 to $p$ with respect to $p$, we have

$$
\begin{aligned}
\psi(p, s)= & \frac{F_{1}(p)}{s}+\frac{F_{2}(p)}{s^{2}}+\frac{F(p, s)}{s^{2}}-\frac{1}{s^{2}} \int_{0}^{p} L_{x} L_{t}\left[\frac{\partial}{\partial x}\left(x \frac{\partial \psi}{\partial x}\right)+x \varphi\right] d p, \\
\varphi(p, s)= & \frac{G_{1}(p)}{s}+\frac{G_{2}(p)}{s^{2}}+\frac{G(p, s)}{s^{2}} \\
& -\frac{1}{s^{2}} \int_{0}^{p} L_{x} L_{t}\left[\frac{\partial}{\partial x}\left(x \frac{\partial \varphi}{\partial x}\right)+x \psi\right] d p .
\end{aligned}
$$

Step 4: Using double Laplace Adomain decomposition methods to define the solution of the system as follows

$$
\psi(x, t)=\sum_{n=0}^{\infty} \psi_{n}(x, t), \quad \varphi(x, t)=\sum_{n=0}^{\infty} \varphi_{n}(x, t) .
$$

Step 5: By operating the inverse transformation of double Laplace on (2.5) and use (2.6), we obtain

$$
\begin{aligned}
\sum_{n=0}^{\infty} \psi_{n}(x, t)= & f_{1}(x)+t f_{2}(x)+L_{p}^{-1} L_{s}^{-1}\left[\frac{F(p, s)}{s^{2}}\right] \\
& -L_{p}^{-1} L_{s}^{-1}\left[\frac{1}{s^{2}} \int_{0}^{p} L_{x} L_{t}\left[\frac{\partial}{\partial x}\left(x \frac{\partial}{\partial x} \sum_{n=0}^{\infty} \psi_{n}\right)\right] d p\right] \\
& -L_{p}^{-1} L_{s}^{-1}\left[\frac{1}{s^{2}} L_{x} L_{t}\left[\int_{0}^{p}\left(x \sum_{n=0}^{\infty} \varphi_{n}\right) d p\right]\right]
\end{aligned}
$$

and

$$
\begin{aligned}
\sum_{n=0}^{\infty} \varphi_{n}(x, t)= & g_{1}(x)+\operatorname{tg}_{2}(x)+L_{p}^{-1} L_{s}^{-1}\left[\frac{G(p, s)}{s^{2}}\right] \\
& -L_{p}^{-1} L_{s}^{-1}\left[\frac{1}{s^{2}} \int_{0}^{p} L_{x} L_{t}\left[\frac{\partial}{\partial x}\left(x \frac{\partial}{\partial x} \sum_{n=0}^{\infty} \varphi_{n}\right)\right] d p\right] \\
& -L_{p}^{-1} L_{s}^{-1}\left[\frac{1}{s^{2}} L_{x} L_{t}\left[\int_{0}^{p}\left(x \sum_{n=0}^{\infty} \psi_{n}\right) d p\right]\right]
\end{aligned}
$$


In particular, we have

$$
\begin{aligned}
& \psi_{0}=f_{1}(x)+\operatorname{tf}_{2}(x)+L_{p}^{-1} L_{s}^{-1}\left[\frac{F(p, s)}{s^{2}}\right], \\
& \varphi_{0}=g_{1}(x)+\operatorname{tg}_{2}(x)+L_{p}^{-1} L_{s}^{-1}\left[\frac{G(p, s)}{s^{2}}\right],
\end{aligned}
$$

and the rest terms can be written as follows

$$
\begin{aligned}
\psi_{n+1}= & -\mathrm{L}_{p}^{-1} \mathrm{~L}_{\mathrm{s}}^{-1}\left[\frac{1}{\mathrm{~s}^{2}} \int_{0}^{\mathrm{p}} \mathrm{L}_{x} \mathrm{~L}_{\mathrm{t}}\left[\frac{\partial}{\partial x}\left(x \frac{\partial}{\partial x} \sum_{n=0}^{\infty} \psi_{n}\right)\right] \mathrm{d} p\right] \\
& -\mathrm{L}_{\mathrm{p}}^{-1} \mathrm{~L}_{\mathrm{s}}^{-1}\left[\frac{1}{\mathrm{~s}^{2}} \mathrm{~L}_{\mathrm{x}} \mathrm{L}_{\mathrm{t}}\left[\int_{0}^{\mathrm{p}}\left(x \sum_{n=0}^{\infty} \varphi_{n}\right) \mathrm{dp}\right]\right],
\end{aligned}
$$

and

$$
\begin{aligned}
\varphi_{n+1}= & -\mathrm{L}_{p}^{-1} \mathrm{~L}_{s}^{-1}\left[\frac{1}{s^{2}} \int_{0}^{p} \mathrm{~L}_{x} \mathrm{~L}_{\mathrm{t}}\left[\frac{\partial}{\partial x}\left(x \frac{\partial}{\partial x} \sum_{n=0}^{\infty} \varphi_{\mathrm{n}}\right)\right] \mathrm{dp}\right] \\
& -\mathrm{L}_{\mathrm{p}}^{-1} \mathrm{~L}_{\mathrm{s}}^{-1}\left[\frac{1}{\mathrm{~s}^{2}} \mathrm{~L}_{\mathrm{x}} \mathrm{L}_{\mathrm{t}}\left[\int_{0}^{\mathrm{p}}\left(x \sum_{\mathrm{n}=0}^{\infty} \psi_{\mathrm{n}}\right) \mathrm{dp}\right]\right] .
\end{aligned}
$$

The above equations (2.7), (2.8) and (2.9) provide an inverse transformation of double Laplace.

In order to confirm our method for solving the singular one dimensional coupled hyperbolic equations, we consider the following example.

Example 2.1. Consider the following nonhomogeneous form of a singular one dimensional system of hyperbolic equations

$$
\begin{aligned}
& \frac{\partial^{2} \psi}{\partial t^{2}}-\frac{1}{x}\left(x \frac{\partial \psi}{\partial x}\right)_{x}-\varphi=-x^{2} \sin t-4 \sin t-x^{2} \cos t \\
& \frac{\partial^{2} \varphi}{\partial t^{2}}-\frac{1}{x}\left(x \frac{\partial \varphi}{\partial x}\right)_{x}-\psi=-x^{2} \cos t-4 \cos t-x^{2} \sin t
\end{aligned}
$$

with the following conditions

$$
\psi(x, 0)=0, \quad \psi_{\mathrm{t}}(x, 0)=x^{2}, \quad \varphi(x, 0)=x^{2}, \quad \varphi_{\mathrm{t}}(x, 0)=0 .
$$

By using the above steps, we obtain

$$
\begin{aligned}
\sum_{n=0}^{\infty} \psi_{n}= & x^{2} \sin t+4 \sin t+x^{2} \cos t-4 t-x^{2} \\
& -L_{p}^{-1} L_{s}^{-1}\left[\frac{1}{s^{2}} \int_{0}^{p} L_{x} L_{t}\left[\left(x\left(\sum_{n=0}^{\infty} \psi_{n x}(x, t)\right)_{x}\right)_{x}\right] d p\right] \\
& -L_{p}^{-1} L_{s}^{-1}\left[\frac{1}{s^{2}} \int_{0}^{p} L_{x} L_{t}\left[\left(\sum_{n=0}^{\infty} \varphi_{n x}(x, t)\right)\right] d p\right]
\end{aligned}
$$

and

$$
\sum_{n=0}^{\infty} \varphi_{n}=x^{2} \cos t+4 \cos t+x^{2} \sin t-4-x^{2} t
$$




$$
\begin{aligned}
& -\mathrm{L}_{\mathrm{p}}^{-1} \mathrm{~L}_{s}^{-1}\left[\frac{1}{\mathrm{~s}^{2}} \int_{0}^{p} \mathrm{~L}_{x} \mathrm{~L}_{\mathrm{t}}\left[\left(x\left(\sum_{n=0}^{\infty} \varphi_{n x}(x, \mathrm{t})\right)_{x}\right)_{x}\right] \mathrm{dp}\right] \\
& -\mathrm{L}_{\mathrm{p}}^{-1} \mathrm{~L}_{\mathrm{s}}^{-1}\left[\frac{1}{\mathrm{~s}^{2}} \int_{0}^{\mathrm{p}} \mathrm{L}_{x} \mathrm{~L}_{\mathrm{t}}\left[\left(\sum_{n=0}^{\infty} \psi_{n x}(x, \mathrm{t})\right)\right] \mathrm{dp}\right] .
\end{aligned}
$$

By using (2.7), (2.8) and (2.9) the components are given by

$$
\begin{aligned}
& \psi_{0}=x^{2} \sin t+4 \sin t+x^{2} \cos t-4 t-x^{2}, \\
& \varphi_{0}=x^{2} \cos t+4 \cos t+x^{2} \sin t-4-x^{2} t,
\end{aligned}
$$

and

$$
\begin{aligned}
\psi_{1} & =-\mathrm{L}_{\mathrm{p}}^{-1} \mathrm{~L}_{s}^{-1}\left[\frac{1}{s^{2}} \int_{0}^{p} \mathrm{~L}_{x} \mathrm{~L}_{\mathrm{t}}\left[\frac{\partial}{\partial x}\left(x \frac{\partial}{\partial x} \psi_{0}\right)+x \varphi_{0}\right] \mathrm{dp}\right] \\
& =\mathrm{L}_{\mathrm{p}}^{-1} \mathrm{~L}_{s}^{-1}\left[\frac{4}{\mathrm{ps}^{2}\left(\mathrm{~s}^{2}+1\right)}+\frac{8}{p s\left(s^{2}+1\right)}+\frac{2}{p^{3} s\left(s^{2}+1\right)}+\frac{4}{p^{3} s^{2}\left(s^{2}+1\right)}-\frac{8}{p s^{3}}-\frac{2}{p^{3} s^{4}}\right], \\
\psi_{1} & =4 t-4 \sin t-8 \cos t+x^{2}-x^{2} \cos t+8+x^{2}+x^{2} t-x^{2} \sin t-4 t^{2}-\frac{1}{6} x^{2} t^{3},
\end{aligned}
$$

and

$$
\begin{aligned}
& \varphi_{1}=-\mathrm{L}_{p}^{-1} \mathrm{~L}_{s}^{-1}\left[\frac{1}{s^{2}} \int_{0}^{p} \mathrm{~L}_{x} \mathrm{~L}_{\mathrm{t}}\left[\frac{\partial}{\partial x}\left(x \frac{\partial}{\partial x} \varphi_{0}\right)+x \psi_{0}\right] \mathrm{dp}\right], \\
& \varphi_{1}=4-8 \sin \mathrm{t}-4 \cos \mathrm{t}+x^{2}-x^{2} \cos \mathrm{t}+8 \mathrm{t}+x^{2}+x^{2} \mathrm{t}-x^{2} \sin \mathrm{t}-\frac{4}{3} \mathrm{t}^{3}-\frac{1}{2} x^{2} \mathrm{t}^{2} .
\end{aligned}
$$

In the same manner, we obtain that

$$
\begin{aligned}
\psi_{2}= & 4 t^{2}+8 \cos t-8+12 \sin t-12 t+2 t^{3}-\frac{1}{10} t^{5} \\
& +\frac{1}{2} x^{2} t^{2}-x^{2} t+x^{2} \cos t-x^{2}+x^{2} \sin t+\frac{1}{6} x^{2} t^{3}-\frac{1}{24} x^{2} t^{4},
\end{aligned}
$$

and

$$
\begin{aligned}
\varphi_{2}= & \frac{4}{3} t^{3}+8 \sin t-8 t+12 \cos t-12+6 t^{2}-\frac{1}{2} t^{4} \\
& +\frac{1}{2} x^{2} t^{2}-x^{2} t+x^{2} \cos t-x^{2}+x^{2} \sin t+\frac{1}{6} x^{2} t^{3}-\frac{1}{120} x^{2} t^{5} .
\end{aligned}
$$

By adding all components, we obtain the exact solution in the following form,

$$
\psi(x, t)=x^{2} \sin t \text { and } \varphi(x, t)=x^{2} \cos t .
$$

\section{Singular nonlinear one dimensional system of hyperbolic equations}

In this part of study, we are concerned about how to use the modified double Laplace method to solve the singular nonlinear one dimensional system of hyperbolic equations which is given by

$$
\begin{aligned}
& \frac{\partial^{2} \psi}{\partial t^{2}}-\frac{1}{x}\left(x \frac{\partial \psi}{\partial x}\right)_{x}-\varphi \frac{\partial \psi}{\partial x}=f(\psi), \\
& \frac{\partial^{2} \varphi}{\partial t^{2}}-\frac{1}{x}\left(x \frac{\partial \varphi}{\partial x}\right)_{x}-\psi \frac{\partial \varphi}{\partial x}=g(\varphi),
\end{aligned}
$$


subject to $(2.2)$ where $f(\psi)$ and $g(\psi)$ are nonlinear functions. In order to get the solution of (3.1), we use the following steps:

Step 1: Multiplying equation (3.1) by $x$.

Step 2: Using Lemma 1.1 and definition of the double Laplace transform of partial derivatives for equations in Step 1 and single Laplace transform for initial condition.

Step 3: Integrating the obtained equations with respect to $p$, from 0 to $p$.

Step 4: By operating the inverse double Laplace transform for equations, we obtain

$$
\begin{aligned}
\psi(x, t)= & f_{1}(x)+t f_{2}(x)-L_{p}^{-1} L_{s}^{-1}\left[\frac{1}{s^{2}} \int_{0}^{p} L_{x} L_{t}[x f(\psi)] d p\right] \\
& -L_{p}^{-1} L_{s}^{-1}\left[\frac{1}{s^{2}} L_{x} L_{t}\left[\int_{0}^{p} \frac{\partial}{\partial x}\left(x \frac{\partial \psi}{\partial x}\right)+x \varphi \frac{\partial \psi}{\partial x} d p\right]\right],
\end{aligned}
$$

and

$$
\begin{aligned}
\varphi(x, t)= & g_{1}(x)+\operatorname{tg}_{2}(x)-L_{p}^{-1} L_{s}^{-1}\left[\frac{1}{s^{2}} \int_{0}^{p} L_{x} L_{t}[x g(\varphi)] d p\right] \\
& -L_{p}^{-1} L_{s}^{-1}\left[\frac{1}{s^{2}} L_{x} L_{t}\left[\int_{0}^{p} \frac{\partial}{\partial x}\left(x \frac{\partial \varphi}{\partial x}\right)+x \psi \frac{\partial \varphi}{\partial x} d p\right]\right]
\end{aligned}
$$

The modified double Laplace decomposition methods (MDLDM) which define the solution of the singular one dimensional system of hyperbolic equations that can be represented as a power series are defined by (2.6). The nonlinear operators can be defined as follows

$$
\mathrm{N}_{1}=\sum_{\mathrm{n}=0}^{\infty} \mathrm{A}_{\mathrm{n}}, \quad \text { and } \quad \mathrm{N}_{2}=\sum_{\mathrm{n}=0}^{\infty} \mathrm{B}_{\mathrm{n}}
$$

where $A_{n}$ and $B_{n}$ are given by

$$
\begin{aligned}
& A_{n}=\frac{1}{n !}\left(\frac{d^{n}}{d \lambda^{n}}\left[N_{1} \sum_{i=0}^{\infty}\left(\lambda^{n} \psi_{n}\right)\right]\right)_{\lambda=0}, \\
& B_{n}=\frac{1}{n !}\left(\frac{d^{n}}{d \lambda^{n}}\left[N_{2} \sum_{i=0}^{\infty}\left(\lambda^{n} \varphi_{n}\right)\right]\right)_{\lambda=0} .
\end{aligned}
$$

Here, Adomain's polynomials $A_{n}$ and $B_{n}$ are given by

$$
\begin{aligned}
& A_{0}=\varphi_{0} \psi_{0 x} \\
& A_{1}=\varphi_{0} \psi_{1 x}+\varphi_{1} \psi_{0 x} \\
& A_{2}=\varphi_{0} \psi_{2 x}+\varphi_{1} \psi_{1 x}+\varphi_{2} \psi_{0 x}
\end{aligned}
$$

and

$$
\begin{aligned}
& \mathrm{B}_{0}=\psi_{0} \varphi_{0 x} \\
& \mathrm{~B}_{1}=\psi_{0} \varphi_{1 \mathrm{x}}+\psi_{1} \varphi_{0 \mathrm{x}} \\
& \mathrm{B}_{2}=\psi_{0} \varphi_{2 \mathrm{x}}+\psi_{1} \varphi_{1 \mathrm{x}}+\psi_{2} \varphi_{0 \mathrm{x}}
\end{aligned}
$$

By substituting (3.4) and (3.5) into (3.2) and (3.3) we obtain

$$
\psi_{0}=f_{1}(x)+t^{2}(x), \quad \varphi_{0}=g_{1}(x)+\operatorname{tg}_{2}(x),
$$


and the rest terms can be written as follows

$$
\begin{aligned}
& \psi_{n+1}=-L_{p}^{-1} L_{s}^{-1}\left[\frac{1}{s^{2}} \int_{0}^{p} L_{x} L_{t}\left[\chi f\left(\sum_{n=0}^{\infty} \psi_{n}\right)\right] d p\right] \\
& -\mathrm{L}_{\mathrm{p}}^{-1} \mathrm{~L}_{\mathrm{s}}^{-1}\left[\frac{1}{\mathrm{~s}^{2}} \int_{0}^{p} \mathrm{~L}_{x} \mathrm{~L}_{\mathrm{t}}\left[\frac{\partial}{\partial x}\left(x \frac{\partial}{\partial x} \sum_{n=0}^{\infty} \psi_{\mathrm{n}}\right)\right] \mathrm{dp}\right] \\
& -\mathrm{L}_{p}^{-1} \mathrm{~L}_{\mathrm{s}}^{-1}\left[\frac{1}{\mathrm{~s}^{2}} \mathrm{~L}_{x} \mathrm{~L}_{\mathrm{t}}\left[\int_{0}^{\mathrm{p}}\left(x \sum_{n=0}^{\infty} A_{n}\right) \mathrm{dp}\right]\right] \text {, }
\end{aligned}
$$

and

$$
\begin{aligned}
& \varphi_{\mathrm{n}+1}=-\mathrm{L}_{\mathrm{p}}^{-1} \mathrm{~L}_{\mathrm{s}}^{-1}\left[\frac{1}{\mathrm{~s}^{2}} \int_{0}^{p} \mathrm{~L}_{\chi} \mathrm{L}_{\mathrm{t}}\left[\chi\left(\sum_{\mathrm{n}=0}^{\infty} \psi_{\mathrm{n}}\right)\right] \mathrm{dp}\right] \\
& -\mathrm{L}_{p}^{-1} \mathrm{~L}_{\mathrm{s}}^{-1}\left[\frac{1}{\mathrm{~s}^{2}} \int_{0}^{p} \mathrm{~L}_{x} \mathrm{~L}_{\mathrm{t}}\left[\frac{\partial}{\partial x}\left(x \frac{\partial}{\partial x} \sum_{n=0}^{\infty} \varphi_{\mathrm{n}}\right)\right] \mathrm{dp}\right] \\
& -\mathrm{L}_{\mathrm{p}}^{-1} \mathrm{~L}_{\mathrm{s}}^{-1}\left[\frac{1}{\mathrm{~s}^{2}} \mathrm{~L}_{x} \mathrm{~L}_{\mathrm{t}}\left[\int_{0}^{\mathrm{p}}\left(x \sum_{\mathrm{n}=0}^{\infty} \mathrm{B}_{\mathrm{n}}\right) \mathrm{dp}\right]\right] \text {. }
\end{aligned}
$$

The inverse Laplace transform is denoted by $\mathrm{L}_{p}^{-1} \mathrm{~L}_{s}^{-1}$ with respect to $p$, s. Here, we provide double inverse Laplace transform with respect to $p$ and $s$ which are given by (3.6) and (3.7).

\section{Convergence analysis of the method}

Finally, we discuss the convergence analysis of the modified double Laplace decomposition methods for the singular nonlinear one dimensional system of hyperbolic equations which is given by

$$
\begin{aligned}
& \frac{\partial^{2} \psi}{\partial \mathrm{t}^{2}}-\frac{1}{x}\left(x \frac{\partial \psi}{\partial x}\right)_{x}-\varphi \frac{\partial \psi}{\partial x}=f(\psi), \\
& \frac{\partial^{2} \varphi}{\partial \mathrm{t}^{2}}-\frac{1}{x}\left(x \frac{\partial \varphi}{\partial x}\right)_{x}-\psi \frac{\partial \varphi}{\partial x}=g(\varphi) .
\end{aligned}
$$

We propose to extend this idea given in [15], for all $\psi, \varphi \in H$. We define $H$ as $H=L_{\mu}^{2}((a, b) \times[0, T])$, $x \in(a, b)$ and

$$
\begin{aligned}
& \psi:(a, b) \times[0, T] \rightarrow \mathbb{R} \times \mathbb{R}, \text { with }\|\psi\|_{H}^{2}=\int_{Q} x \psi^{2}(x, t) d x d t \\
& (\psi, \varphi)=\int_{Q} x \psi(x, t) \varphi(x, t) d x d t
\end{aligned}
$$

where $\mathrm{Q}=(\mathrm{a}, \mathrm{b}) \times[0, \mathrm{~T}]$ and

$$
H=\left\{\begin{array}{c}
(\psi, \varphi):(a, b) \times[0, T], \text { with } \\
L_{p}^{-1} L_{s}^{-1}\left[\frac{1}{s^{2}} \int_{0}^{p} L_{x} L_{t}[\psi(x, t)](p, s) d p\right](x, t)<\infty
\end{array}\right\} .
$$

Multiply (4.1) by $x$, and write the equation in the operator form as follows

$$
\begin{aligned}
& L(\psi)=x \frac{\partial^{2} \psi}{\partial t^{2}}=\frac{\partial \psi}{\partial x}+x \frac{\partial \psi}{\partial x}+x \varphi \frac{\partial \psi}{\partial x}+x f(\psi), \\
& L(\varphi)=x \frac{\partial^{2} \varphi}{\partial t^{2}}=\frac{\partial \varphi}{\partial x}+x \frac{\partial^{2} \varphi}{\partial x^{2}}+x \psi \frac{\partial \varphi}{\partial x}+x g(\varphi) .
\end{aligned}
$$

For $\mathrm{L}$ is hemicontinuous operator, consider the following hypotheses: 
(H1) $(\mathrm{L}(\psi)-\mathrm{L}(w), \psi-w) \geqslant \mathrm{k}\|\psi-w\|^{2}$ and $(\mathrm{L}(\varphi)-\mathrm{L}(w), \varphi-w) \geqslant \mathrm{k}\|\varphi-w\|^{2} ; \quad \mathrm{k}>0, \forall \psi, \varphi, w \in \mathrm{H}$;

(H2) whatever may be $M^{*}>0$, there exists a constant $C\left(M^{*}\right)>0$ such that for $\psi, w \in H$ with $\|\psi\| \leqslant$ $M^{*},\|\varphi\| \leqslant M^{*},\|w\| \leqslant M^{*}$ we have

$$
(\mathrm{L}(\psi)-\mathrm{L}(w), z) \leqslant \mathrm{C}\left(M^{*}\right)\|\psi-z\|\|w\|, \text { and }(\mathrm{L}(\varphi)-\mathrm{L}(z), w) \leqslant \mathrm{C}\left(M^{*}\right)\|\varphi-z\|\|w\|,
$$

for every $w, z \in \mathrm{H}$.

In the next theorem we follow [9-11].

Theorem 4.1 (Sufficient condition of convergence). The Modified double Laplace decomposition methods applied to the singular nonlinear one dimensional system of hyperbolic equations (4.2) without initial and boundary conditions, converges towards a particular solution.

Proof. First, we check the hypothesis (H1) for the operator $\mathrm{L}(\psi), \mathrm{L}(\varphi)$ of $(4.2)$. we use the definition of our operator $\mathrm{L}$, and then we have

$$
\begin{aligned}
L(\psi)-L(w)= & \left(\frac{\partial \psi}{\partial x}-\frac{\partial w}{\partial x}\right)+\left(x \frac{\partial \psi}{\partial x}-x \frac{\partial w}{\partial x}\right) \\
& +\left(x \varphi \frac{\partial \psi}{\partial x}-x \varphi \frac{\partial w}{\partial x}\right)+x(f(\psi)-f(w)) \\
= & (\psi-w)_{x}+x \frac{\partial^{2}}{\partial x^{2}}(\psi-w) \\
& +x \varphi \frac{\partial}{\partial x}(\psi-w)+x(f(\psi)-f(w))
\end{aligned}
$$

and

$$
\begin{aligned}
L(\varphi)-L(w)= & \left(\frac{\partial \varphi}{\partial x}-\frac{\partial w}{\partial x}\right)+\left(x \frac{\partial^{2} \varphi}{\partial x^{2}}-x \frac{\partial w}{\partial x}\right) \\
& +\left(x \psi \frac{\partial \varphi}{\partial x}-x \psi \frac{\partial w}{\partial x}\right)+x(g(\varphi)-f(w)) \\
= & \frac{\partial}{\partial x}(\varphi-w)+x \frac{\partial^{2}}{\partial x^{2}}(\varphi-w) \\
& +x \psi \frac{\partial}{\partial x}(\varphi-w)+x(g(\varphi)-f(w))
\end{aligned}
$$

therefore,

$$
\begin{aligned}
(L(\psi)-L(w), \psi-w)= & \left(\frac{\partial}{\partial x}(\psi-w), \psi-w\right) \\
& +\left(x \frac{\partial^{2}}{\partial x^{2}}(\psi-w), \psi-w\right) \\
& +\left(x \varphi \frac{\partial}{\partial x}(\psi-w), \psi-w\right) \\
& +(x(f(\psi)-f(w)), \psi-w)
\end{aligned}
$$

and

$$
\begin{aligned}
(\mathrm{L}(\varphi)-\mathrm{L}(w), \varphi-w)= & \left(\frac{\partial}{\partial x}(\varphi-w), \varphi-w\right) \\
& +\left(x \frac{\partial^{2}}{\partial x^{2}}(\varphi-w), \varphi-w\right) \\
& +\left(x \psi \frac{\partial}{\partial x}(\varphi-w), \varphi-w\right) \\
& +(x(g(\varphi)-f(w)), \varphi-w)
\end{aligned}
$$


According to the coercive operator, $\frac{\partial}{\partial x}$ and $\frac{\partial^{2}}{\partial x^{2}}$ in $H$, then there exist constants $\alpha, \beta, \delta>0$ such that

$$
\left(\frac{\partial}{\partial x}(\psi-w), \psi-w\right) \geqslant \alpha\|\psi-w\|^{2}
$$

and

$$
\begin{aligned}
-\left(x \frac{\partial^{2}}{\partial x^{2}}(\psi-w), \psi-w\right) & \leqslant|x|\left\|(\psi-w)_{x x}\right\|\|\psi-w\| \\
& \leqslant b \beta\|\psi-w\|^{2}, \\
& \Leftrightarrow \\
\left(x \frac{\partial^{2}}{\partial x^{2}}(\psi-w), \psi-w\right) & \geqslant-b \beta\|\psi-w\|^{2},
\end{aligned}
$$

where $\|\psi\| \leqslant M^{*},\|\varphi\| \leqslant M^{*},\|w\| \leqslant M^{*}$, and according to the Schwarz inequality, we get

$$
\begin{aligned}
-\left(x \varphi \frac{\partial}{\partial x}(\psi-w), \psi-w\right) & \leqslant|x|\|\varphi\|\left\|(\psi-w)_{x}\right\|\|\psi-w\| \\
& \leqslant \mathrm{bM}^{*} \delta\|\psi-\varphi\|\|\psi-\varphi\| \\
& \leqslant \mathrm{bM}^{*} \delta\|\psi-w\|^{2} \\
& \leqslant \mathrm{~b} \delta M^{*}\|\psi-w\|^{2}
\end{aligned}
$$

hence,

$$
\left(x \varphi \frac{\partial}{\partial x}(\psi-w), \psi-w\right) \geqslant-b \delta M^{*}\|\psi-w\|^{2} .
$$

By using Cauchy-Schwarz inequality, where $\sigma>0$ and $\mathrm{f}$ is Lipschitzian function, we have

$$
\begin{aligned}
(-x(f(\psi)-f(w)), \psi-w) & \leqslant|x|\|f(\psi)-f(w)\|\|\psi-w\| \\
& \leqslant b\|f(\psi)-f(w)\|\|\psi-w\| \\
& \leqslant b \sigma\|\psi-w\|^{2} \\
& \Leftrightarrow \\
(x(f(\psi)-f(w)), \psi-\varphi) & \geqslant-b \sigma\|\psi-w\|^{2} .
\end{aligned}
$$

Substituting (4.5), (4.6), (4.8) and (4.9) into equation (4.3) gives

$$
\begin{aligned}
& (\mathrm{L}(\psi)-\mathrm{L}(w), \psi-w) \geqslant\left(\alpha-\mathrm{b} \beta-\mathrm{b} \delta \mathrm{M}^{*}-\mathrm{b} \sigma\right)\|\psi-w\|^{2}, \\
& (\mathrm{~L}(\psi)-\mathrm{L}(w), \psi-w) \geqslant k\|\psi-w\|^{2},
\end{aligned}
$$

where

$$
k=\alpha-b \beta-b \delta M^{*}-b \sigma>0 .
$$

By the same method for (4.4) there exist constants $\zeta, \eta, \lambda, \rho>0$ such that

$$
\begin{aligned}
& (\mathrm{L}(\varphi)-\mathrm{L}(w), \varphi-w) \geqslant\left(\zeta-\mathrm{b} \eta-\mathrm{b} \lambda \mathrm{M}^{*}-\mathrm{b} \rho\right)\|\varphi-w\|^{2}, \\
& (\mathrm{~L}(\varphi)-\mathrm{L}(w), \varphi-w) \geqslant \mathrm{k}_{1}\|\varphi-w\|^{2}
\end{aligned}
$$

where

$$
k_{1}=\zeta-b \eta-b \lambda M^{*}-b \rho>0 .
$$


So the first part (H1) is checked. Now we are going to prove $(\mathrm{H} 2)$ for the operator $\mathrm{L}(\psi)$ and $\mathrm{L}(\varphi)$. For every $M^{*}>0$, there exists a constant $C\left(M^{*}\right)>0$ such that for $\psi, \varphi, w \in H$ with $\|\psi\| \leqslant M^{*},\|\varphi\| \leqslant M^{*}$,

$$
\left(\mathrm{L}(\psi)-\mathrm{L}(w), z_{1}\right) \leqslant \mathrm{C}\left(\mathrm{M}^{*}\right)\|\psi-w\|\left\|z_{1}\right\|,
$$

for every $z_{1}, z_{2} \in \mathrm{H}$. Therefore we have,

$$
\begin{aligned}
\left(\mathrm{L}(\psi)-\mathrm{L}(w), z_{1}\right)= & \left((\psi-w)_{x}, z_{1}\right) \\
& +\left(x \frac{\partial^{2}}{\partial x^{2}}(\psi-w), z_{1}\right) \\
& +\left(x \varphi \frac{\partial}{\partial x}(\psi-w), z_{1}\right) \\
& +\left(x(f(\psi)-f(w)), z_{1}\right) .
\end{aligned}
$$

By using the Cauchy-Schwartz inequality and the fact that $\psi$ and $w$ are bounded, we obtain the following

$$
\begin{gathered}
\left(\frac{\partial}{\partial x}(\psi-w), z_{1}\right) \leqslant \alpha_{1}\|\psi-w\|\left\|z_{1}\right\|, \\
\left(x \frac{\partial^{2}}{\partial x^{2}}(\psi-w), z_{1}\right) \leqslant \mathrm{b} \beta_{1}\|\psi-w\|\left\|z_{1}\right\|, \\
\left(x \varphi \frac{\partial}{\partial x}(\psi-w), z_{1}\right) \leqslant \alpha_{2}|x|\|\varphi\|\|\psi-w\|\left\|z_{1}\right\| \\
\leqslant b \alpha_{2} M^{*}\|\psi-w\|\left\|z_{1}\right\|,
\end{gathered}
$$

and

$$
\left(x(f(\psi)-f(w)), z_{1}\right) \leqslant b \sigma_{1}\|\psi-w\|\left\|z_{1}\right\| .
$$

For the constants $\alpha_{1}, \beta_{1}, \alpha_{2}, \sigma_{1}>0$, we have:

$$
\begin{aligned}
\left(\mathrm{L}(\psi)-\mathrm{L}(w), z_{1}\right) & \leqslant\left(\alpha_{1}+\mathrm{b} \beta_{1}+\mathrm{b} \alpha_{2} M^{*}+\mathrm{b} \sigma_{1}\right)\|\psi-w\|\left\|z_{1}\right\| \\
& =\mathrm{C}\left(M^{*}\right)\|\psi-w\|\left\|z_{1}\right\|,
\end{aligned}
$$

where

$$
C\left(M^{*}\right)=\left(\alpha_{1}+b \beta_{1}+b \alpha_{2} M^{*}+b \sigma_{1}\right),
$$

and

$$
\begin{aligned}
\left(\mathrm{L}(\varphi)-\mathrm{L}(w), z_{2}\right)= & \left(\frac{\partial}{\partial x}(\varphi-w), z_{2}\right) \\
& +\left(x \frac{\partial^{2}}{\partial x^{2}}(\varphi-w), z_{2}\right) \\
& +\left(x \psi \frac{\partial}{\partial x}(\varphi-w), z_{2}\right) \\
& +\left(x(g(\varphi)-f(w)), z_{2}\right)
\end{aligned}
$$

Similarly, we get,

$$
\begin{aligned}
\left(\mathrm{L}(\varphi)-\mathrm{L}(w), z_{2}\right) & \leqslant\left(\zeta_{1}+\mathrm{b} \eta_{1}+\mathrm{b} \lambda_{1} M^{*}+\mathrm{b} \rho_{1}\right)\|\varphi-w\|\left\|z_{2}\right\| \\
& =\mathrm{C}\left(M^{*}\right)\|\varphi-w\|\left\|z_{2}\right\|,
\end{aligned}
$$

where $C\left(M^{*}\right)=\zeta_{1}+b \eta_{1}+b \lambda_{1} M^{*}+b \rho_{1}$ and $\zeta_{1}, \eta_{1}, \lambda_{1}, \rho_{1}>0$. Therefore (H2) holds. 
Conclusion 4.2. A combination of double Laplace transform and decomposition method has been used in this paper to seek a solution of the linear and nonlinear singular one dimensional system of hyperbolic equations. Second, we presented a convergence proof of the (DLADM) applied to the nonlinear singular one dimensional system of hyperbolic equations.

\section{Acknowledgment}

The author would like to extend his sincere appreciation to the Deanship of Scientific Research at King Saud University for its funding of this research through the Research Group Project number RGP-117.

\section{References}

[1] K. Abbaoui, Y. Cherruault, Convergence of Adomian's method applied to differential equations, Comput. Math. Appl., 28 (1994), 103-109. 1

[2] K. Abbaoui, Y. Cherruault, Convergence of Adomian's method applied to nonlinear equations, Math. Comput. Modelling, 20 (1994), 69-73. 1

[3] F. M. Allan, K. Al-Khaled, An approximation of the analytic solution of the shock wave equation, J. Comput. Appl. Math., 192 (2006), 301-309. 1

[4] A. Atangana, S. C. Oukouomi Noutchie, On multi-Laplace transform for solving nonlinear partial differential equations with mixed derivatives, Math. Probl. Eng., 2014 (2014), 9 pages. 1

[5] E. Babolian, J. Biazar, On the order of convergence of Adomian method, Appl. Math. Comput., 130 (2002), 383-387. 1

[6] Y. Cherruault, G. Saccomandi, B. Some, New results for convergence of Adomian's method applied to integral equations, Math. Comput. Modelling, 16 (1992), 85-93. 1

[7] S. M. El-Sayed, D. Kaya, On the numerical solution of the system of two-dimensional Burgers' equations by the decomposition method, Appl. Math. Comput., 158 (2004), 101-109. 1

[8] H. Eltayeb, A. K1lıçman, A note on solutions of wave, Laplace's and heat equations with convolution terms by using a double Laplace transform, Appl. Math. Lett., 21 (2008), 1324-1329. 1

[9] I. Hashim, M. S. M. Noorani, M. R. Said Al-Hadidi, Solving the generalized Burgers-Huxley equation using the Adomian decomposition method, Math. Comput. Modelling, 43 (2006), 1404-1411. 4

[10] D. Kaya, I. E. Inan, A convergence analysis of the ADM and an application, Appl. Math. Comput., 161 (2005), 10151025.

[11] D. Kaya, I. E. Inan, A numerical application of the decomposition method for the combined KdVMKdV equation, Appl. Math. Comput., 168 (2005), 915-926. 4

[12] S. A. Khuri, A Laplace decomposition algorithm applied to a class of nonlinear differential equations, J. Appl. Math., 1 (2001), 141-155. 1

[13] A. Kıliçman, H. Eltayeb, A note on defining singular integral as distribution and partial differential equations with convolution term, Math. Comput. Modelling, 49 (2009), 327-336. 1

[14] A. Kılıçman, H. E. Gadain, On the applications of Laplace and Sumudu transforms, J. Franklin Inst., 347 (2010), 848 862. 1

[15] T. Mavoungou, Y. Cherruault, Numerical study of Fisher's equation by Adomian's method, Math. Comput. Modelling, 19 (1994), 89-95. 4

[16] S. S. Ray, A numerical solution of the coupled sine-Gordon equation using the modified decomposition method, Appl. Math. Comput., 175 (2006), 1046-1054. 1

[17] N. H. Sweilam, Harmonic wave generation in non linear thermoelasticity by variational iteration method and Adomian's method, J. Comput. Appl. Math., 207 (2007), 64-72. 1

[18] E. Yusufoğlu, Numerical solution of Duffing equation by the Laplace decomposition algorithm, Appl. Math. Comput., 177 (2006), 572-580. 1 